PROCEEDINGS OF THE

AMERICAN MATHEMATICAL SOCIETY

Volume 128, Number 4, Pages 1125-1134

S 0002-9939(99)05208-9

Article electronically published on August 5, 1999

\title{
BOUNDEDNESS OF INTEGRAL OPERATORS ON GENERALIZED MORREY SPACES AND ITS APPLICATION TO SCHRÖDINGER OPERATORS
}

\author{
KAZUHIRO KURATA, SEIICHI NISHIGAKI, AND SATOKO SUGANO \\ (Communicated by Christopher D. Sogge)
}

\begin{abstract}
In this paper, we study boundedness of integral operators on generalized Morrey spaces and its application to estimates in Morrey spaces for the Schrödinger operator $L_{2}=-\Delta+V(x)+W(x)$ with nonnegative $V \in(R H)_{\infty}$ (reverse Hölder class) and small perturbed potentials $W$.
\end{abstract}

\section{INTRODUCTION}

In this paper, we study boundedness of integral operators with the kernel of Riesz type on generalized Morrey spaces (Theorems 2.1, 2.2) and its application to the study of mapping properties of the Schrödinger operator $L_{2}=-\Delta+V(x)+W(x)$ with nonnegative $V \in(R H)_{\infty}$ (reverse Hölder class) and small perturbed potentials $W$ (Theorems 1.2, 1.3, 3.1, 3.2). We say $V \in(R H)_{\infty}$ if there exists a constant $C$ such that

$$
\sup _{y \in B(x, r)}|V(y)| \leq C \frac{1}{|B(x, r)|} \int_{B(x, r)}|V(y)| d y
$$

for every $x \in \mathbf{R}^{n}$ and $r>0$, where $B(x, r)=\left\{y \in \mathbf{R}^{n} ;|y-x|<r\right\}$. As a typical example, for any polynomial $P(x)$ and $\alpha>0, V(x)=|P(x)|^{\alpha}$ belongs to this class. For $L_{1}=-\Delta+V, V \in(R H)_{\infty}, V \not \equiv 0$, we already know nice estimates for the fundamental solution $\Gamma(x, y)$ for $L_{1}$ due to Z. Shen [Sh1]: for every $k>0$ there exists a constant $C_{k}$ such that

$$
\begin{aligned}
|\Gamma(x, y)| & \leq \frac{C_{k}}{(1+m(x, V)|x-y|)^{k}|x-y|^{n-2}}, \\
\left|\nabla_{x} \Gamma(x, y)\right|+\left|\nabla_{y} \Gamma(x, y)\right| & \leq \frac{C_{k}}{(1+m(x, V)|x-y|)^{k}|x-y|^{n-1}},
\end{aligned}
$$

where $m(x, V)$ is defined by

$$
\frac{1}{m(x, V)}=\sup \left\{r>0 ; \frac{r^{2}}{|B(x, r)|} \int_{B(x, r)} V(y) d y \leq 1\right\} .
$$

We note that $V \in(R H)_{\infty}$ satisfies $V(x) \leq C m(x, V)^{2}$ for some constant $C$. For further properties of $m(x, V)$, we refer to [Sh1]. The estimates (1.2)-(1.3) enable

Received by the editors June 1, 1998.

1991 Mathematics Subject Classification. Primary 35B45, 42B20; Secondary 35J10.

The first author was partially supported by Grant-in Aid for Scientific Research (C)(No. 09640208), the Ministry of Education, Science, Sports and Culture. 
us to obtain the boundedness of $L_{1}^{-1}$, the integral operator with the kernel $\Gamma(x, y)$, on various spaces. In particular, we can prove that there exists a constant $C$ such that

$$
\left|m(x, V)^{2}\left(L_{1}^{-1} f\right)(x)\right|+\left|m(x, V) \nabla L_{1}^{-1} f(x)\right| \leq C M(|f|)(x)
$$

for $f \in L_{c}^{\infty}\left(\mathbf{R}^{n}\right)$, where $M(|f|)$ is the Hardy-Littlewood maximal function of $f$ and $L_{c}^{\infty}\left(\mathbf{R}^{n}\right)=\left\{f \in L^{\infty}\left(\mathbf{R}^{n}\right)\right.$; supp $f$ is compact $\}$ (see [KS] and Lemma 3.1). The main purpose of this paper is to show estimates of $L_{2}^{-1}$ (see Definition 1.2) for certain perturbed potentials $W$ (not necessarily nonnegative) on various spaces. We should remark that, if we consider an example $W(x)=-\epsilon /|x|^{2}$, we cannot expect such nice estimates for the fundamental solution to $L_{2}$. Our method relies on the estimates for $L_{1}$ and a simple perturbation argument. To develop a perturbation theory, we consider integral operators $T$ and $S_{M}$ defined by

$$
T f(x)=\int_{\mathbf{R}^{n}} \frac{f(y)}{|x-y|^{n / s}} d y
$$

and

$$
S_{M} f(x)=\int_{\mathbf{R}^{n}} \frac{f(y)}{|x-y|^{n / s}(1+|x-y|)^{M}} d y
$$

for some $s>1$ and $M>0$. Many authors studied the boundedness of $T$ on various spaces, e.g., on $L^{p}$ spaces, weighted $L^{p}$ spaces, and classical Morrey spaces. Among them, we note the recent result due to Olsen, which covers all previous results, except for weighted $L^{p}$ spaces (see, e.g., $[\mathrm{Ad}],[\mathrm{CF}]$, [Ta]). We recall that a function $f$ is said to belong to the classical Morrey space $M_{p}^{r}, 1 \leq p \leq r$, if

$$
\|f\|_{M_{p}^{r}} \equiv \sup _{Q \subset \mathbf{R}^{n}}|Q|^{1 / r-1 / p}\left(\int_{Q}|f(x)|^{p} d x\right)^{1 / p}<+\infty
$$

holds, where the supremum is taken over all cubes $Q \subset \mathbf{R}^{n}$. For a function $W(x)$, we denote by $W \cdot T$ the product of $W$ and $T$, where $W$ is the multiplication operator by $W(x)$. The following theorem is due to Olsen [Ol], although Olsen stated the estimate only in the three-dimensional case.

Theorem 1.1 ([О] $)$. Let $n \geq 3$ and $1 / v+1 / s=1$. Assume $1<p \leq r, p<u \leq$ $v, 1 / r+1 / s>1$, and $W \in M_{u}^{v}$. Then there exists a constant $C$ such that

$$
\|W \cdot T f\|_{M_{p}^{r}} \leq C\|W\|_{M_{u}^{v}}\|f\|_{M_{p}^{r}}, \quad f \in M_{p}^{r} .
$$

We assume that $V$ satisfies the following conditions: there exists a positive constant $m_{0}$ such that

$$
V(x) \geq 0, V \in(R H)_{\infty}, m(x, V) \geq m_{0} .
$$

For example, $V(x)=|P(x)|^{\alpha}$ satisfies (1.8) for every polynomials $P(x)$ and $\alpha>0$ (see [Sh1]).

Definition 1.1. For a Banach space $Y\left(\subset L_{l o c}^{p}\left(\mathbf{R}^{n}\right)\right.$ for some $\left.1<p<+\infty\right)$ we consider the Banach space $\mathcal{X}_{Y}$ associated with $Y$ :

$$
\mathcal{X}_{Y}=\left\{u \in Y ; m(x, V)^{2} u, m(x, V) \nabla u, \nabla^{2} u \in Y\right\}
$$

with its norm $\|u\|_{\mathcal{X}_{Y}}=\left\|m(x, V)^{2} u\right\|_{Y}+\|m(x, V) \nabla u\|_{Y}+\left\|\nabla^{2} u\right\|_{Y}$.

Let $L_{1}=-\Delta+V$ and $L_{2}=L_{1}+W$. Now, we state the estimate in the classical Morrey spaces for $L_{2}$. 
Theorem 1.2. Assume $n \geq 3,1<p \leq r<n / 2, p<u \leq n / 2$, and (1.8) for $V$. Let $Y=M_{p}^{r}$ and suppose $W \in M_{u}^{n / 2}$ and $\|W\|_{M_{u}^{n / 2}}$ is sufficiently small. Then there exists a constant $C$ such that

$$
\begin{aligned}
& \|W u\|_{M_{p}^{r}}+\left\|m(x, V)^{2} u\right\|_{M_{p}^{r}}+\|m(x, V) \nabla u\|_{M_{p}^{r}}+\left\|\nabla^{2} u\right\|_{M_{p}^{r}} \\
& \leq C\left\|L_{2} u\right\|_{M_{p}^{r}}, \quad u \in C_{0}^{\infty}\left(\mathbf{R}^{n}\right) .
\end{aligned}
$$

Here $C$ depends on $\|W\|_{M_{u}^{n / 2}}$ and $\left\|L_{1}^{-1}\right\|_{Y \rightarrow \mathcal{X}_{Y}}$.

For the definition of $\left\|L_{1}^{-1}\right\|_{Y \rightarrow \mathcal{X}_{Y}}$, see section 3

Definition 1.2. $\left(L_{2}^{-1}\right)$ Under the assumption that $W L_{1}^{-1}$ is bounded on $Y$ and $W$ is small in the sense that $\left\|W L_{1}^{-1}\right\|_{Y \rightarrow Y}<1$, we define the operator $L_{2}^{-1}$ from $Y$ to $\mathcal{X}_{Y}$ by $L_{2}^{-1}=L_{1}^{-1}\left(1+W L_{1}^{-1}\right)^{-1}$. Note that, when $L_{1}$ is an isomorphism from $\mathcal{X}_{Y}$ to $Y, u=L_{2}^{-1} f \in \mathcal{X}_{Y}$ represents a unique solution to $L_{2} u=f$ for $f \in Y$.

Since we will see that $L_{1}$ gives an isomorphism from $\mathcal{X}$ to $Y$ for the case $Y=$ $L^{p}\left(\mathbf{R}^{n}\right)$ with $1<p<+\infty$, we have the following theorem.

Theorem 1.3. (1) Assume $n \geq 3,1<p<n / 2, p<u \leq n / 2$, and (1.8) for $V$. Suppose $W \in M_{u}^{n / 2}$ and $\|W\|_{M_{u}^{n / 2}}$ is sufficiently small. Then there exists a constant $C$ such that

$$
\begin{aligned}
& \left\|W L_{2}^{-1} f\right\|_{L^{p}}+\left\|m(x, V)^{2} L_{2}^{-1} f\right\|_{L^{p}}+\left\|m(x, V) \nabla L_{2}^{-1} f\right\|_{L^{p}}+\left\|\nabla^{2} L_{2}^{-1} f\right\|_{L^{p}} \\
& \quad \leq C\|f\|_{L^{p}}, \quad f \in L^{p} .
\end{aligned}
$$

(2) Under the additional assumption $|W|^{1 / 2} \in M_{v}^{n}$ for some $v \leq n$, there exists a constant $C$ such that

$$
\left\||W|^{1 / 2} \nabla L_{2}^{-1} f\right\|_{L^{p}} \leq C\|f\|_{L^{p}}, \quad f \in L^{p} .
$$

Example 1.1. Let $W(x)=\epsilon /|x|^{2}$ with $|\epsilon| \leq \epsilon_{0}$ for sufficiently small $\epsilon_{0}>0$. Then $W \in M_{u}^{n / 2}$ for every $u<n / 2$ and $\|W\|_{M_{u}^{n / 2}} \leq \epsilon_{0} C$ for some constant $C$ which depends only on $n$. Hence we can apply Theorem 1.2 and Theorem 1.3 for every $1<p \leq r<n / 2$ and $1<p<n / 2$, respectively. Note that $W$ does not belong to any $L^{u}$ spaces.

For a general weight function $\Phi(x, r) \geq 0$, the generalized Morrey space $L_{p}^{\Phi}$ with $1 \leq p<+\infty$ is defined as follows:

$$
L_{p}^{\Phi}=\left\{f \in L_{l o c}^{p}\left(\mathbf{R}^{n}\right) ; \sup _{x \in \mathbf{R}^{n}, r>0} \frac{1}{\Phi(x, r)} \int_{B(x, r)}|f(y)|^{p} d y \equiv\|f\|_{p, \Phi}^{p}<+\infty\right\} .
$$

In [Mi and [Na], the authors studied the boundedness of the Hardy-Littlewood maximal function and singular integral operators on those spaces under a certain conditions on $\Phi((2.1)$ and (2.2) in section 2). We should mention that for the restricted case $p / r \leq u / v$, the estimate in Theorem 1.1 has been proved in $\mathrm{CF}$ and its proof is rather simple compared with the one in [Ol]. Since this type of estimate is useful even for the restricted case for our application, we shall generalize it to the generalized Morrey spaces in section 2 under a certain additional condition (2.3) on $\Phi(x, r)$ (Theorem [2.1). Furthermore, we improve the estimate in Theorem 2.1] for the intergral operator $S_{M}$ (Theorem[2.2). In section 3 we apply it to the Schrödinger operator and show a similar estimate as in Theorem 1.2 on the generalized Morrey 
space (Theorems 3.1 3.2). The proof of Theorem 1.2 is also given in a similar way as in Theorem 3.1 .

\section{Boundedness of INTEGRAL OPERATORS ON GENERALIZED MORREY SPACES}

In this section, we show boundedness of the integral operators $T$ and $S_{M}$ on generalized Morrey spaces. We assume that the weight function $\Phi(x, r)$ satisfies the following conditions: there exist a positive constant $C$ and nonnegative constants $\lambda, \delta$ with $\lambda+\delta<n\left(1-p / s^{\prime}\right)$ such that

$$
\begin{aligned}
& C^{-1} \leq \frac{\Phi(x, t)}{\Phi(x, r)} \leq C \quad(r \leq t \leq 2 r), \\
& \int_{r}^{\infty} \frac{\Phi(x, t)}{t^{n-\left(n / s^{\prime}\right) p}} \frac{d t}{t} \leq C \frac{\Phi(x, r)}{r^{n\left(1-p / s^{\prime}\right)}}, \\
& \Phi(x, r) \leq C r^{\lambda}\left(1+r^{\delta}\right)
\end{aligned}
$$

for every $x \in \mathbf{R}^{n}$ and $r>0$, where $s^{\prime}=s /(s-1)$.

Example 2.1. It is easy to see that $\Phi(x, r)=r^{\lambda} \log (2+r)$ with $0 \leq \lambda<n\left(1-p / s^{\prime}\right)$ satisfies the conditions above for every $\delta>0$. Note that $L_{p}^{\Phi}$ does not belong to any classical Morrey spaces.

We state our main results for boundedness of $W \cdot T$ on generalized Morrey spaces.

Theorem 2.1. Assume $n \geq 3,1<s, 1<p<s^{\prime}$, and (2.1)-(2.3) for $\Phi(x, r)$. Let $\lambda^{*}=n /(n-\lambda),(\lambda+\delta)^{*}=n /(n-(\lambda+\delta))$ and $W \in L_{s^{\prime} / \lambda^{*}}^{\Phi} \cap L_{s^{\prime} /(\lambda+\delta)^{*}}^{\Phi}$. Then there exists a constant $C$ such that

$$
\|W \cdot T f\|_{p, \Phi} \leq C\left(\|W\|_{s^{\prime} / \lambda^{*}, \Phi}+\|W\|_{s^{\prime} /(\lambda+\delta)^{*}, \Phi}\right)\|f\|_{p, \Phi}, \quad f \in L_{p}^{\Phi} .
$$

Remark 2.1. Note that, for the case $\Phi(x, t)=t^{n(1-p / r)}$ we have $L_{p}^{\Phi}=M_{p}^{r}$. Hence Theorem 2.1] in the case $\delta=0$ is a generalization of [CF, Theorem 2].

For the integral operator $S_{M}$, we can improve the estimate in Theorem 2.1

Theorem 2.2. Assume $n \geq 3,1<s, 1<p<s^{\prime}$, and (2.1)-(2.3) for $\Phi(x, r)$. Let $W \in L_{s^{\prime}}^{\Phi}$ and suppose $M \geq(\lambda+\delta) / p$. Then there exists a constant $C$ such that

$$
\left\|W \cdot S_{M} f\right\|_{p, \Phi} \leq C\|W\|_{s^{\prime}, \Phi}\|f\|_{p, \Phi}, \quad f \in L_{p}^{\Phi} .
$$

In [Na, Theorem 3], without the assumption (2.3), Nakai proved a boundedness of $T$ from $L_{p}^{\Phi}$ to $L_{q}^{\Phi^{q / p}}$, where $1 / q=1 / p-1 / s^{\prime}$ which is a generalization of [CF] Corollary, p. 277].

Theorem 2.3 ( $\mathbb{N a})$. Suppose $1<p<s^{\prime}$ and $\Phi(x, r)$ satisfies the conditions (2.1) and (2.2). Let $1 / q=1 / p-1 / s^{\prime}$. Then there exists a constant $C$ such that

$$
\|T f\|_{q, \Phi^{q / p}} \leq C\|f\|_{p, \Phi} .
$$

We note Hölder's inequality on the generalized Morrey spaces.

Lemma 2.1. Let $\Phi_{1}(x, r)$ and $\Phi_{2}(x, r)$ be weight functions. Suppose $p, q, t \in(0,+\infty)$ satisfy $1 / t=1 / p+1 / q$ and let $\Psi(x, r)=\Phi_{1}(x, r)^{t / p} \Phi_{2}(x, r)^{t / q}$. Then we have

$$
\|f g\|_{t, \Psi} \leq\|f\|_{p, \Phi_{1}}\|g\|_{q, \Phi_{2}}, \quad f \in L_{p}^{\Phi_{1}}, g \in L_{q}^{\Phi_{2}} .
$$


Proof. For each $x \in \mathbf{R}^{n}$ and $r>0$, we have

$$
\begin{aligned}
\left(\int_{B(x, r)}|f g|^{t} d y\right)^{1 / t} & \leq\left(\int_{B(x, r)}|f|^{p} d y\right)^{1 / p}\left(\int_{B(x, r)}|g|^{q} d y\right)^{1 / q} \\
& \leq\|f\|_{p, \Phi_{1}} \Phi_{1}(x, r)^{1 / p}\|g\|_{q, \Phi_{2}} \Phi_{2}(x, r)^{1 / q} \\
& =\|f\|_{p, \Phi_{1}}\|g\|_{q, \Phi_{2}} \Psi(x, r)^{1 / t} .
\end{aligned}
$$

This implies the desired inequality.

We need the boundedness of $W \cdot T$ on the same generalized Morrey space to apply a perturbation argument to the operator $L_{2}=L_{1}+W$. From this point of view, $W$ must belong to some $L^{q}$ space if we apply Theorem 2.3 .

Corollary 2.1. Suppose $1<p<s^{\prime}$ and $\Phi(x, r)$ satisfies the conditions (2.1) and (2.2). Let $W \in L^{s^{\prime}}\left(\mathbf{R}^{n}\right)$. Then there exists a constant $C$ such that

$$
\|W \cdot T f\|_{p, \Phi} \leq C\|W\|_{L^{s^{\prime}}}\|f\|_{p, \Phi} .
$$

Proof. It is an easy consequence of Theorem 2.3 and Lemma 2.1.

Corollary 2.1 should be compared with Theorem 2.2 (or 2.1). The difference between Theorem 2.2 and Corollary 2.1 can be seen in Example 3.1. for example. To prove Theorem 2.1 we need the following lemma.

Lemma 2.2 ([Na). Under the assumptions (2.1)-(2.2), there exist positive constants $C$ and $\mu$ such that

$$
\int_{r}^{+\infty} \frac{\Phi(x, t)}{t^{n\left(1-p / s^{\prime}\right)-\mu}} \frac{d t}{t} \leq C \frac{\Phi(x, r)}{r^{n\left(1-p / s^{\prime}\right)-\mu}}
$$

holds for every $r>0$.

Proof of Theorem [2.1. We may assume $f \in L_{p}^{\Phi} \cap L_{c}^{\infty}$ without loss of generality in the proof. Actually, we show there exists a constant $C$ which does not depend on $f$ such that

$$
\int_{B(y, r)}|W(x)(T|f|)(x)|^{p} d y \leq C \Phi(y, r)\left(\|W\|_{s^{\prime} / \lambda^{*}, \Phi}+\|W\|_{s^{\prime} /(\lambda+\delta)^{*}, \Phi}\right)^{p}\|f\|_{p, \Phi}^{p}
$$

for every $y \in \mathbf{R}^{n}$ and $r>0$. For general $f \in L_{p}^{\Phi}$, applying (2.6) to $f_{R}(x)=$ $\chi_{\{|x| \leq R\} \cap\{x ;|f(x)| \leq R\}}(x)|f|(x)$ which belongs to $L_{c}^{\infty} \cap L_{p}^{\Phi}$, we have

$$
\int_{B(y, r)}\left|W(x)\left(T\left|f_{R}\right|\right)(x)\right|^{p} d y \leq C \Phi(y, r)\left(\|W\|_{s^{\prime} / \lambda^{*}, \Phi}+\|W\|_{s^{\prime} /(\lambda+\delta)^{*}, \Phi}\right)^{p}\|f\|_{p, \Phi}^{p}
$$

for every $y \in \mathbf{R}^{n}$ and $r>0$. Taking the limit $R \rightarrow \infty$, by the monotone convergence theorem, we obtain (2.6) for general $f \in L_{p}^{\Phi}$ and complete the proof.

(STEP 1): We follow the argument in CF. We write, for $\epsilon>0$ which will be determined later,

$$
T f(x)=\int_{\{|x-y|<\epsilon\}} \frac{|f(y)|}{|x-y|^{n / s}} d y+\int_{\{|x-y| \geq \epsilon\}} \frac{|f(y)|}{|x-y|^{n / s}} d y \equiv I_{1}+I_{2} .
$$


We can estimate $I_{1}$ by

$$
\left|I_{1}\right| \leq \sum_{k=-\infty}^{-1} \int_{\left\{2^{k} \epsilon \leq|x-y|<2^{k+1} \epsilon\right\}} \frac{|f(y)|}{|x-y|^{n / s}} d y \leq C \epsilon^{n(1-1 / s)} M f(x)
$$

for some constant $C$. Now, we take $\sigma$ to satisfy $n\left(1-\frac{p}{s^{\prime}}\right)-\mu<\sigma<p n\left(\frac{1}{s}-\frac{1}{p^{\prime}}\right)$, where $\mu>0$ is the constant in Lemma 2.2 Since $n / s=\sigma / p+\{-(\sigma / p-n / s)\}$, Hölder's inequality yields

$$
\left|I_{2}\right| \leq\left(\int_{\{|x-y| \geq \epsilon\}} \frac{|f(y)|^{p}}{|x-y|^{\sigma}} d y\right)^{1 / p}\left(\int_{\{|x-y| \geq \epsilon\}}|x-y|^{(\sigma / p-n / s) p^{\prime}} d y\right)^{1 / p^{\prime}} \equiv I_{3} I_{4} .
$$

For $I_{3}$, we have by (2.1)

$$
\begin{aligned}
I_{3} & \leq\left(\sum_{k=0}^{+\infty} \int_{\left\{2^{k} \epsilon \leq|x-y|<2^{k+1} \epsilon\right\}} \frac{|f(y)|^{p}}{\left(2^{k} \epsilon\right)^{\sigma}} d y\right)^{1 / p} \\
& \leq\left(\sum_{k=0}^{+\infty} \frac{C \Phi\left(x, 2^{k} \epsilon\right)}{\left(2^{k} \epsilon\right)^{\sigma}}\right)^{1 / p}\|f\|_{p, \Phi .}
\end{aligned}
$$

We obtain

$$
\begin{aligned}
\sum_{k=0}^{+\infty} \frac{\Phi\left(x, 2^{k} \epsilon\right)}{\left(2^{k} \epsilon\right)^{\sigma}} & =\sum_{k=0}^{+\infty} \frac{\left(2^{k} \epsilon\right)^{n\left(1-p / s^{\prime}\right)-\mu}}{\left(2^{k} \epsilon\right)^{\sigma}} \frac{C \Phi\left(x, 2^{k} \epsilon\right)}{\left(2^{k} \epsilon\right)^{n\left(1-p / s^{\prime}\right)-\mu}} \\
& \leq C \sum_{k=0}^{+\infty} \frac{\left(2^{k} \epsilon\right)^{n\left(1-p / s^{\prime}\right)-\mu}}{\left(2^{k} \epsilon\right)^{\sigma}} \int_{2^{k} \epsilon}^{2^{k+1} \epsilon} \frac{\Phi(x, t)}{t^{n\left(1-p / s^{\prime}\right)-\mu}} \frac{d t}{t} \\
& \leq C \frac{\epsilon^{n\left(1-p / s^{\prime}\right)-\mu}}{\epsilon^{\sigma}} \int_{\epsilon}^{\infty} \frac{\Phi(x, t)}{t^{n\left(1-p / s^{\prime}\right)-\mu}} \frac{d t}{t}
\end{aligned}
$$

for some constant $C$. Here, since $\sigma>n\left(1-p / s^{\prime}\right)-\mu$, we used $\left(2^{k}\right)^{n\left(1-p / s^{\prime}\right)-\mu-\sigma} \leq 1$ for every $k \geq 0$. By Lemma 2.2, we can conclude

$$
\sum_{k=0}^{+\infty} \frac{\Phi\left(x, 2^{k} \epsilon\right)}{\left(2^{k} \epsilon\right)^{\sigma}} \leq C \frac{\Phi(x, \epsilon)}{\epsilon^{\sigma}} .
$$

On the other hand, it is easy to obtain

$$
I_{4} \leq C \epsilon^{n\left(1 / p^{\prime}-1 / s\right)+\sigma / p} .
$$

Hence, it follows that

$$
I_{2} \leq C \Phi(x, \epsilon)^{1 / p} \epsilon^{n(1-1 / p-1 / s)}\|f\|_{p, \Phi} .
$$

Therefore, we obtain that

$$
(T f)(x) \leq C\left\{\epsilon^{n(1-1 / s)} M f(x)+\Phi(x, \epsilon)^{1 / p} \epsilon^{n(1-1 / p-1 / s)}\|f\|_{p, \Phi}\right\}
$$

holds for some constant $C$. 
(STEP 2): By the condition (2.3), we have

$$
(T f)(x) \leq C\left\{\epsilon^{n(1-1 / s)} M f(x)+\left(\epsilon^{\lambda / p}+\epsilon^{(\lambda+\delta) / p}\right) \epsilon^{n(1-1 / p-1 / s)}\|f\|_{p, \Phi}\right\} .
$$

Let $A=M(|f|)(x)$ and $B=\|f\|_{p, \Phi}$. We consider two cases.

Case 1. For the case $B / A \leq 1$, i.e. $\|f\|_{p, \Phi} \leq M(|f|)(x)$, we choose $\epsilon=(B / A)^{p /(n-\lambda)}$. Since $\epsilon \leq 1$, it follows that

$$
\begin{aligned}
(T f)(x) & \leq C\left\{\left(\frac{B}{A}\right)^{\frac{n p(1-1 / s)}{n-\lambda}} A+\left(\frac{B}{A}\right)^{\frac{p}{n-\lambda}(\lambda / p+n(1-1 / p-1 / s))} B\right\} \\
& \leq C M(|f|)(x)^{1-p \lambda^{*} / s^{\prime}}\|f\|_{p, \Phi}^{p \lambda^{*} / s^{\prime}} .
\end{aligned}
$$

Case 2. For the case $B / A \geq 1$, i.e. $\|f\|_{p, \Phi} \geq M(|f|)(x)$, we choose

$$
\epsilon=(B / A)^{p /(n-(\lambda+\delta))} \text {. }
$$

In this case, it follows in a similar way that

$$
(T f)(x) \leq C M(|f|)(x)^{1-p(\lambda+\delta)^{*} / s^{\prime}}\|f\|_{p, \Phi}^{p(\lambda+\delta)^{*} / s^{\prime}}
$$

Hence, we obtain

$$
\begin{aligned}
(T f)(x) & \leq C M(|f|)(x)^{1-p \lambda^{*} / s^{\prime}}\|f\|_{p, \Phi}^{p \lambda^{*} / s^{\prime}}+C M(|f|)(x)^{1-p(\lambda+\delta)^{*} / s^{\prime}}\|f\|_{p, \Phi}^{p(\lambda+\delta)^{*} / s^{\prime}} \\
& \equiv J_{1}(x)+J_{2}(x) .
\end{aligned}
$$

Define $a \in(1, \infty)$ by $1 / a=1-p \lambda^{*} / s^{\prime}$. By using Hölder's inequality, we have

$$
\begin{aligned}
& \left(\int_{B(y, r)}\left|W(x) J_{1}(x)\right|^{p} d y\right)^{1 / p} \\
& \quad \leq C\left(\int_{B(y, r)}|M f(x)|^{p / a}|W(x)|^{p} d y\right)^{1 / p}\|f\|_{p, \Phi}^{1 / a^{\prime}} \\
& \quad \leq C\left(\int_{B(y, r)}|M f(x)|^{p} d y\right)^{1 / a p}\left(\int_{B(y, r)}|W(x)|^{s^{\prime} / \lambda^{*}} d y\right)^{\lambda^{*} / s^{\prime}}\|f\|_{p, \Phi}^{1 / a^{\prime}} \\
& \leq C \Phi(y, r)^{1 / p}\|W\|_{s^{\prime} / \lambda^{*}, \Phi}\|f\|_{p, \Phi .}
\end{aligned}
$$

In the last inequality we used the fact that $\|M f\|_{p, \Phi} \leq C\|f\|_{p, \Phi}$ holds under the assumptions (2.1) and (2.2) (see $\mathrm{Na}]$ ). This implies

$$
\left\|W J_{1}\right\|_{p, \Phi} \leq C\|W\|_{s^{\prime} / \lambda^{*}, \Phi}\|f\|_{p, \Phi}
$$

In a similar way we have

$$
\left\|W J_{2}\right\|_{p, \Phi} \leq C\|W\|_{s^{\prime} /(\lambda+\delta)^{*}, \Phi}\|f\|_{p, \Phi} .
$$

This complete the proof of Theorem 2.1.

Proof of Theorem 2.2. We modify the proof of Theorem 2.1] in the following. We can estimate $I_{2}$ by $I_{3}^{\prime}$ and $I_{4}$, where

$$
I_{3^{\prime}}=\left(\int_{\{|x-y| \geq \epsilon\}} \frac{|f(y)|^{p}}{|x-y|^{\sigma}(1+|x-y|)^{M p}} d y\right)^{1 / p} .
$$


We can estimate $I_{3^{\prime}}$ in two different ways, namely $I_{3^{\prime}} \leq I_{3}$ and

$$
\begin{aligned}
I_{3^{\prime}} & \leq\left(\sum_{k=0}^{\infty} \int_{\left\{2^{k} \epsilon \leq|x-y|<2^{k+1} \epsilon\right\}} \frac{|f(y)|^{p}}{|x-y|^{\sigma+M p}} d y\right)^{1 / p} \\
& \leq C \frac{\Phi(x, r)^{1 / p}}{\epsilon^{M}} \epsilon^{n(1-1 / p-1 / s)}\|f\|_{p, \Phi .}
\end{aligned}
$$

Therefore, we obtain

$$
\begin{aligned}
(T f)(x) & \leq C \epsilon^{n(1-1 / s)} M(f)(x)+C \epsilon^{\lambda / p} \min \left(1+\epsilon^{\delta / p}, \frac{1+\epsilon^{\delta / p}}{\epsilon^{M}}\right) \epsilon^{n(1-1 / p-1 / s)}\|f\|_{p, \Phi} \\
& \leq C \epsilon^{n(1-1 / s)} M(f)(x)+C \epsilon^{n(1-1 / p-1 / s)}\|f\|_{p, \Phi .}
\end{aligned}
$$

Here we used the boundedness of $\epsilon^{\lambda / p} \min \left(1+\epsilon^{\delta / p}, \frac{1+\epsilon^{\delta / p}}{\epsilon^{M}}\right)$ under the assumption $M \geq(\lambda+\delta) / p$. Thus, this yields

$$
(T f)(x) \leq C(M f)(x)^{1-p / s^{\prime}}\|f\|_{p, \Phi}^{p / s^{\prime}}
$$

which completes the proof of Theorem 2.2

\section{Application to Schrödinger operators}

We consider Schrödinger operators $L_{1}=-\Delta+V(x)$ and $L_{2}=L_{1}+W(x)$ on $\mathbf{R}^{n}, n \geq 3$. We study estimates in Morrey spaces and boundedness of $L_{2}^{-1}$ for certain small perturbed potentials $W$ by using estimates for the fundamental solution for $L_{1}^{-1}$.

Lemma $3.1([\mathrm{KS}])$. Suppose $V(x) \geq 0, V \in(R H)_{\infty}$. Then there exist constants $C_{j}, j=1,2$, such that

$$
\left|m(x, V)^{2} L_{1}^{-1} f(x)\right| \leq C_{1} M(f)(x),\left|m(x, V) \nabla L_{1}^{-1} f(x)\right| \leq C_{2} M(f)(x),
$$

for $f \in L_{c}^{\infty}\left(\mathbf{R}^{n}\right)$.

Let $Y\left(\subset L_{l o c}^{p}\left(\mathbf{R}^{n}\right)\right.$ for some $\left.1<p<+\infty\right)$ be a Banach space, and let $\mathcal{X}_{Y}$ be the Banach space associated with $Y$ in Definition 1.1.

Corollary 3.1. (1) Suppose $V(x) \geq 0, V \in(R H)_{\infty}$ and that there exists a constant $C$ such that $\|M(f)\|_{Y}+\left\|\nabla^{2}(-\Delta)^{-1} f\right\|_{Y} \leq C\|f\|_{Y}$ for $f \in Y \cap L_{c}^{\infty}$. Then there exists a constant $C_{1}$ such that

$$
\left\|L_{1}^{-1} f\right\|_{\mathcal{X}_{Y}} \leq C_{1}\|f\|_{Y}, \quad f \in Y \cap L_{c}^{\infty} .
$$

(2) Under the same assumptions, there exists a constant $C_{2}$ such that

$$
\|u\|_{\mathcal{X}_{Y}} \leq C_{2}\left\|L_{1} u\right\|_{Y}, \quad u \in C_{0}^{\infty}\left(\mathbf{R}^{n}\right) .
$$

Proof. Lemma 3.1 and the assumption yield

$$
\left\|m(x, V)^{2} L_{1}^{-1} f\right\|_{Y}+\left\|m(x, V) \nabla L_{1}^{-1} f\right\|_{Y}+\left\|\Delta L_{1}^{-1} f\right\|_{Y} \leq C\|f\|_{Y} .
$$

Since $\nabla^{2} L_{1}^{-1}=\nabla^{2}(-\Delta)^{-1}(-\Delta) L_{1}^{-1}$, we obtain (1). Since $L_{1} u=f \in L_{c}^{\infty} \cap Y$ for $u \in C_{0}^{\infty}\left(\mathbf{R}^{n}\right)$ and $u=L_{1}^{-1} f,(2)$ is a consequence of $(1)$. 
We can show in the same way as in the proof of Theorem[2.1 that

$$
\left\|m(x, V)^{2} L_{1}^{-1} f\right\|_{Y}+\left\|m(x, V) \nabla L_{1}^{-1} f\right\|_{Y} \leq C\|f\|_{Y}
$$

holds for $f \in Y$. We denote by $\left\|L_{1}^{-1}\right\|_{Y \rightarrow \mathcal{X}_{Y}}$ the infimum of the constant $C_{2}$ in Corollary 3.1 Since it is known that the assumption in Corollary 3.1 holds for

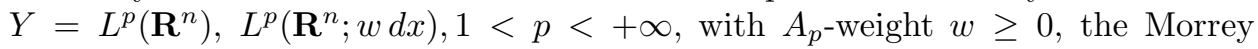
spaces $M_{p}^{r}, 1<p \leq r<\infty$, generalized Morrey spaces $L_{p}^{\Phi}$ (see, e.g., [CF], [GR], [Mi], [Na]), we can apply Corollary 3.1 to these Banach spaces $Y$. Now we state our main result in this section.

Theorem 3.1. Assume $n \geq 3,1<p<n / 2,0 \leq \lambda+\delta<n(1-2 p / n)$, (1.8) for $V$, and that $\Phi(x, r)$ satisfies the conditions (2.1)-(2.3). Let $Y=L_{p}^{\Phi}$ and $W \in L_{n / 2}^{\Phi}$. Suppose $\|W\|_{n / 2, \Phi}$ is sufficiently small. Then there exists a constant $C$ such that

$$
\|W u\|_{Y}+\|u\|_{\mathcal{X}_{Y}} \leq C\left\|L_{2} u\right\|_{Y}, \quad u \in C_{0}^{\infty}\left(\mathbf{R}^{n}\right) .
$$

Here $C$ depends on $\|W\|_{L_{n / 2, \Phi}}$ and $\left\|L_{1}^{-1}\right\|_{Y \rightarrow \mathcal{X}_{Y}}$.

Proof. It is known that the fundamental solution $\Gamma(x, y)$ for $L_{1}$ satisfies $(1.2)$ and (1.3) and hence

$$
0 \leq \Gamma(x, y) \leq \frac{C_{M}}{|x-y|^{n-2}(1+|x-y|)^{M}}
$$

for every $M>0$ under the assumption (1.8) on $V$. Thus $W L_{1}^{-1}$ is a bounded operator on $Y$ under the assumption by Theorem 2.2 with $s=n /(n-2)$ and hence $\left(1+W L_{1}^{-1}\right)^{-1}$ is also bounded on $Y$ because of the smallness of $\|W\|_{n / 2, \Phi}$ and can be written as Neumann series. For $u \in C_{0}^{\infty}\left(\mathbf{R}^{n}\right), L_{2} u=f$ is equivalent to $L_{1} u=\left(1+W L_{1}^{-1}\right)^{-1} f$. Then, the desired estimate is an easy consequence of Theorem 2.2 with $s=n /(n-2)$ and Corollary $3.1(2)$.

Proof of Theorem 1.2. If we combine Theorem 1.1 with $s=n /(n-2)$ and the estimate $|\Gamma(x, y)| \leq C|x-y|^{2-n}$, we can prove Theorem 1.2 in the same way as in the proof of Theorem 3.1

Under somewhat weaker assumptions that $V$ satisfies $V(x) \geq 0, V \not \equiv 0$, and $V \in(R H)_{\infty}$, if we apply Theorem 2.1 instead of Theorem [2.2] we can obtain a similar estimate as in Theorem 3.1. If we apply Corollary 2.1, we have

Theorem 3.2. Assume $n \geq 3,1<p<n / 2, V(x) \geq 0, V(x) \not \equiv 0, V \in(R H)_{\infty}$, and $\Phi(x, r)$ satisfies the conditions (2.1) and (2.2). Let $Y=L_{p}^{\Phi}, W \in L^{n / 2}\left(\mathbf{R}^{n}\right)$. Suppose $\|W\|_{L^{n / 2}}$ is sufficiently small. Then there exists a constant $C$ such that

$$
\|W u\|_{Y}+\|u\|_{\mathcal{X}_{Y}} \leq C\left\|L_{2} u\right\|_{Y}, \quad u \in C_{0}^{\infty}\left(\mathbf{R}^{n}\right) .
$$

Example 3.1. We compare Theorem 3.1 with Theorem 3.2 in the case $\Phi(x, r)=$ $\log (2+r)$, for example. Hence, $\Phi(x, r)$ satisfies (2.1)-(2.3) with $\lambda=0$ and small $\delta>0$. Note that $L^{n / 2}\left(\mathbf{R}^{n}\right) \subset L_{n / 2}^{\Phi}$. Thus Theorem 3.1 is stronger than Theorem 3.2 in this case. For the case $\Phi(x, r)=r^{\lambda} \log (2+r)$ with $0<\lambda<n(1-2 p / n)$ and small $\delta>0$, Theorem 3.1 and Theorem 3.2 complement each other.

Proof of Theorem 1.3. By Corollary 3.1 (2) there exists a constant $C$ such that

$$
\left\|m(x, V)^{2} u\right\|_{L^{p}}+\|m(x, V) \nabla u\|_{L^{p}}+\left\|\nabla^{2} u\right\|_{L^{p}} \leq C\left\|L_{1} u\right\|_{L^{p}}
$$

for every $u \in C_{0}^{\infty}\left(\mathbf{R}^{n}\right)$. On the other hand, Kato's inequality implies the operator $L_{1}$ with the domain $D\left(L_{1}\right)=C_{0}^{\infty}\left(\mathbf{R}^{n}\right)$ is essentially $m$-accretive in $L^{p}$ with $1<$ 
$p<+\infty$ (see, e.g., $[\mathrm{He}],[\mathrm{Ok}],[\mathrm{Ku}]$ ). Combining these results, we obtain that the closure of $L_{1}$ is an isomorphism from $\mathcal{X}_{Y}$ to $Y=L^{p}\left(\mathbf{R}^{n}\right)$ (see also $[\mathrm{Gu}$ ). Hence, $L_{2}^{-1} f=L_{1}^{-1}\left(1+W L_{1}^{-1}\right)^{-1} f$ represents a unique solution of $L_{2} u=f$ for $f \in L^{p}\left(\mathbf{R}^{n}\right)$. Theorem 1.3(1) is a consequence of Theorem 1.1] with $s=n / 2$ and Theorem 1.2. Under the assumptions on $V$, we have

$$
\left|\nabla_{x} \Gamma(x, y)\right| \leq \frac{C_{M}}{(1+|x-y|)^{M}|x-y|^{n-1}}
$$

for every $M>0$. Hence Theorem 1.1 with $s=n$ yields the boundedness of $|W|^{1 / 2} \nabla L_{1}^{-1}$ on $L^{p}\left(\mathbf{R}^{n}\right)$. Thus it follows that

$$
\begin{aligned}
\left\||W|^{1 / 2} \nabla L_{2}^{-1} f\right\|_{L^{p}} & =\left\||W|^{1 / 2} \nabla L_{1}^{-1}\left(1+W L_{1}^{-1}\right)^{-1} f\right\|_{L^{p}} \\
& \leq C\left\|\left(1+W L_{1}^{-1}\right)^{-1} f\right\|_{L^{p}} \leq C\|f\|_{L^{p}} .
\end{aligned}
$$

Theorem 1.3) (2) is a consequence of Theorem 1.1] with $s=n$.

We should mention that our method can also be applied to other operators, e.g., uniformly elliptic operators and magnetic Schrödinger operators ([KS], [Sh2]).

\section{REFERENCES}

[Ad] D. Adams, A note on Riesz potentials, Duke Math. J. 42(1975), 765-778.

[CF] F.Chiarenza, M.Frasca, Morrey spaces and Hardy-Littlewood maximal function, Rend. Mat. 7(1987), 273-279. MR 90f:42017

[GR] J. García-Cuerva, J. L. Rubio de Francia, Weighted Norm Inequalities and Related Topics, North-Holland, 1985.

[Gu D.Guibourg, Inégalitës maximales pour l'opérateur de Schrödinger, C.R. Acad.Sci.Paris, 316(1993), 249-252. MR 93k:35053

[He] D.Henry, Geometric Theory of semilinear parabolic equations, Springer Lect. Note, No. 840, 1981. MR 83j:35084

[Ku] S.T.Kuroda, Spectral Theory II(in Japanese), Iwanami-Shoten, 1979. MR 88a:35169

[KS] K.Kurata, S.Sugano, A remark on estimates for uniformly elliptic operators on weighted $L^{p}$ spaces and Morrey spaces, preprint.

[Mi] T. Mizuhara, Boundedness of some classical operators on generalized Morrey spaces, Harmonic Analysis (S.Igari, Ed.) ICM 90 Satellite Proceedings, Springer-Verlag, Tokyo(1991), 183-189. MR 95c:46039

[Na] E. Nakai, Hardy-Littlewood maximal operator, singular integral operators and the Riesz potentials on generalized Morrey spaces, Math. Nachr. 166(1994), 95-103. MR 95k:42030

[Ok] N. Okazawa, On the perturbation of linear operators in Banach and Hilbert spaces, J.Math.Soc.Japan, 34, 1982, 677-701. MR 84i:47021

[Ol] P.A.Olsen, Fractional Integration, Morrey spaces and a Schrödinger equations, Comm. in P.D.E., 20(1995), 2005-2055. MR 97a:35042

[Sh1] Z.Shen, $L^{p}$ estimates for Schrödinger operators with certain potentials, Ann. Inst. Fourier, Grenoble 45, 2(1995), 513-546. MR 96h:35037

[Sh2] Z.Shen, Estimates in $L^{p}$ for Magnetic Schrödinger Operators, Indiana Univ. Math. J., 45(1996), 817-841. MR 97k:35043

[Ta] M.Taylor, Microlocal Analysis on Morrey spaces, Preprint.

Department of Mathematics, Tokyo Metropolitan University, 1-1 Minami-Ohsawa, HACHIOJI-SHI, TOKYO 192-0397, JAPAN

E-mail address: kurata@comp.metro-u.ac.jp

Numazu College of Technology, 3600 Ooka Numazu 410-8501, Japan

E-mail address: nishiga@la.numazu-ct.ac.jp

Department of Mathematics, Gakushuin University, 1-5-1 Mejiro, toshima-ku, Tokyo 171, JAPAN

E-mail address: 95243001@gakushuin.ac.jp 